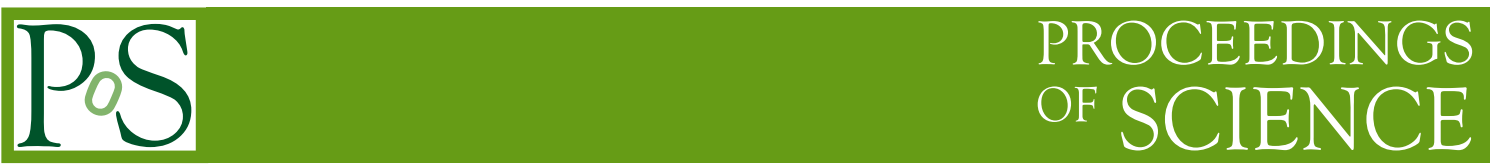

\title{
From galactic stellar populations to the EBL
}

\author{
Alberto Franceschini*i \\ Univ. Padova, Italy \\ E-mail: alberto.franceschini@unipd.it
}

We review our previous determinations of the EBL photon density in the Universe and its evolution with cosmic time (Franceschini et al. 2008, henceforth AF2008), in the light of new recent observations at long wavelengths. These new data indicate slightly lower photon densities longwards of a few microns, in the mid- and far-infrared and sub-millimeter. This implies slightly lower cosmic opacity for photon-photon interactions, that reduces some tension that had emerged in the interpretation of the highest energy TeV observations of local BLAZARs. Few applications of this new modeling of the EBL are briefly discussed.

Science with the New Generation of High Energy Gamma-ray experiments, 10th Workshop 04-06 June 2014

Lisbon - Portugal

\footnotetext{
*Speaker.

${ }^{\dagger}$ More details about the content of this paper will be reported shortly in a dedicated more extensive publication.
} 


\section{Introduction}

The extragalactic background radiation at various electro-magnetic frequencies, from radio to gamma-rays, is a fundamental constituent of the Universe, and was demonstrated to permeate it quite uniformly. Such radiations have a key role during most of the history of universal expansion and the formation of all structures. Given their obliquity, radiations are a fundamental source of opacity for the propagation of high-energy cosmic-ray particles and photons throughout space-time (Nikishov 1962, Gould and Schreder 1966).

One particularly important component of this radiation is the Extragalactic Background Light (EBL), in the wavelength interval between 0.1 and 1000 microns: interactions with these of very high-energy photons from astrophysical sources, and the consequent pair production, produce strong and observable opacity effects (e.g Stecker et al. 1992, among many others). The corresponding high-energy exponential cutoffs are customarily identified in the $\mathrm{TeV}$ spectra of several of the most local BLAZARs observed with imaging atmospheric Cherenkov telescopes, now operating between a few tens of $\mathrm{GeV}$ to tens of TeV (e.g. HEGRA, HESS, MAGIC, see e.g. Dwek and Slavin 1994, Stanev and Franceschini 1998, Aharonian et al. 2006, Albert et al. 2006). Of course, not only photons from cosmic sources interact with EBL photons, but any particle of sufficiently high energy, from cosmic rays to neutrinos. So the issue of an high-precision determination of the time-evolving EBL is of central importance.

Several efforts to modeling the EBL and its time evolution have been published, including those based on physical prescriptions about the formation and evolution of cosmic structures, in particular the semi-analytical models of galaxy formation. This is the so-called forward evolution approach, advocated by Gilmore et al. (2012), among others. The alternative are the more empirical approaches essentially based on local properties of cosmic sources and educated extrapolations back in cosmic time (e.g. Stanev \& Franceschini 1998, Stecker et al. 2006, AF2008, Stecker, Malkan \& Scully 2006), the backward evolution modeling.

Once the background photon densities are defined, the calculation of the cosmic photon-photon opacity and its effects on very high-energy spectra of BLAZARs are standard physical practice (e.g Stecker et al. 1992). Many papers have compared the predictions for such attenuated spectra with multi-wavelength BLAZAR observations, and the results appear often rather consistent, for both local and high-redshift objects. As a particularly interesting and illustrative example of what it is currently feasible, Ackermann et al. (2012) analyze years of Fermi LAT observations of a large sample of BLAZARs over a wide range of redshifts to investigate the effects of pair-production opacities in various redshift bins, assuming smooth spectral extrapolations towards the highest energies and the EBL evolutionary models by AF2008. These LAT normalized BLAZAR spectra show indeed cut-off features increasing with redshift exactly as expected by the model, so providing remarkable proof of the overall validity of our understanding of the local EBL photon densities and their evolution in time.

Similarly, the multi-wavelength high-energy spectra of both BL Lac and Flat-spectrum radio quasars (the AGN populations emitting at the highest energies) are usually fairly well reproduced in terms of standard BLAZAR emission models (like the Synchrotron Self Compton or the External Compton processes), once corrected for photon-photon absorption based on the most accurate EBL models. 
There are however still some areas of concern related with observations of the highest energy BLAZAR spectra. One is the reported relative independence (or only moderate dependence) of the observed spectral indexes of BLAZARs in the limited redshift interval currently probed at $\mathrm{TeV}$ energies, while a faster increase might be expected due to the strong dependence of the opacity on the source distance (De Angelis et al. 2009, Persic \& de Angelis 2008). Another related consideration was about the detection of variable emission in the energy range 70 to $400 \mathrm{GeV}$ from the flat spectrum radio quasar PKS 1222+216, where such high-energy photons would be expected to be absorbed in the broad line region (Tavecchio et al. 2012).

Another source of possible concern are some apparent upturns of the spectra of BLAZARs at the highest energies, once the observed spectra are corrected for EBL absorption. One characteristic instance would be shown by the HEGRA observations of Mkn 501, whose EBL-corrected very high energy spectrum would show a statistically very significant hardening above $10 \mathrm{TeV}$, as reported for example in Costamante (2012).

All the above considerations may have important implications for fundamental physics, as they have raised an important question about photon propagation in space. It has been suggested that photons can oscillate into axion-like particles (ALPs), which are a generic prediction of extensions of the Standard Model of elementary particles, in particular the super-string theories. Photons can oscillate with axions in a similar way as massive neutrinos do, which would require an external field, e.g. a nano-Gauss magnetic field, to compensate for the photon and ALP spin difference. Then a fraction of very-high-energy photons could escape absorption, because ALPs do not interact with ambient photons (De Angelis et al. 2007, Tavecchio et al. 2012). ALPs may be an important constituent of the cosmos because, while not contributing to the cold dark matter, they are a possible candidate for the quintessential dark energy.

The present paper is dedicated to reconsider the issue of the propagation in space-time of the highest energy cosmic photons produced by BLAZARs. In particular, we update a model of the EBL by AF2008 by including new very extensive data in the far-IR and sub-millimeter obtained with the Herschel space observatory, in a spectral region where the EBL and its evolution with time cannot be directly measured. The updated model makes also full use of deep survey data from the Spitzer telescope.

Section 2 summarizes the new data. Section 3 reports on the updated EBL model and consequent modification of the cosmic photon-photon opacities. Section 4 shows applications to the analysis of some BLAZAR spectra.

We use in the following a standard cosmological environment with $H_{0}=70 \mathrm{~km} \mathrm{~s}^{-1} \mathrm{Mpc}^{-1}$, $\Omega_{m}=0.3, \Omega_{\Lambda}=0.7$.

\section{The New Data}

The new data made available during the recent years for estimating the contributions of extragalactic sources to the EBL concern in particular the source infrared emission. Instead, our knowledge of the optical and near-IR part of galaxy emissivities has largely remained unmodified. Indeed the cosmic opacities originating from UV-optical background photons estimated by AF2008 have proven so far rather successful in reproducing the BLAZAR spectra at about $1 \mathrm{TeV}$ or less. 
The new data at the longer wavelengths have mostly come from two great infrared observatories in space, the NASA Spitzer telescope and the ESA Herschel observatory.
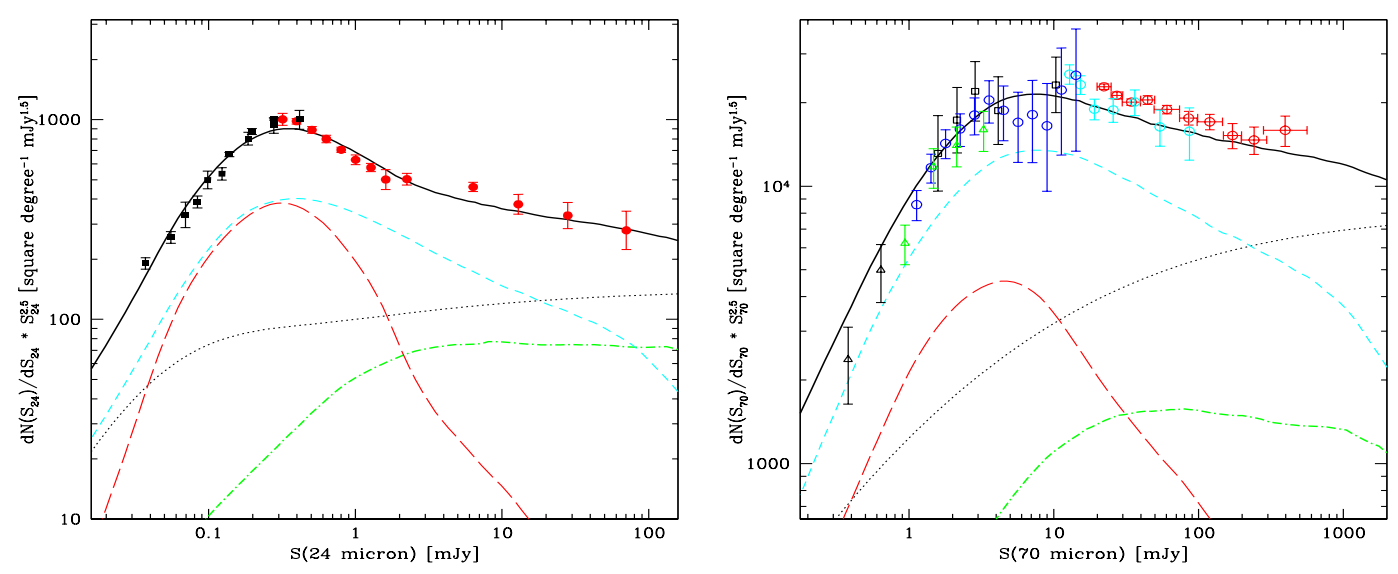

Figure 1: Left: Euclidean-normalized differential number counts of extragalactic sources at $24 \mu \mathrm{m}$ compared with our model fit. The red circles are from the analysis of SWIRE survey data by Shupe et al. (2008), black squares from Papovich et al. (2004). Right: Euclidean-normalized differential number counts of extragalactic sources at $24 \mu \mathrm{m}$. The blue open circles are from Herschel observations by Berta et al. (2011), black triangles from a stacking analysis of Spitzer data by Bethermin et al. (2010). Other data-points are as reported in Franceschini et al. (2010). The contribution by type-I AGNs is shown as green dot-dashed line, moderate-luminosity starbursts (the LIRGs) make the cyan short-dash line (type-II AGNs and starbursts are included in the same population on the assumption that in both classes the IR spectrum is dominated by starburst emission). The red long-dashed line corresponds to the population of high-luminosity sources dominating the IR emissivity at high redshifts. The dotted line is the separate contribution of normal spirals, while the continuous line is the total model counts.

\subsection{The Spitzer MIPS number counts and luminosity functions}

The Spitzer MIPS imaging camera has been used to map deep sky regions in the $24 \mu \mathrm{m}$ channel and partly at $70 \mu \mathrm{m}$. The former observations benefited by excellent sensitivity and good spatial resolution, while the latter observations were limited in both senses. From the local EBL view-point, what matters particularly are the source number counts at both wavelengths, that are reported in differential normalized Euclidean units in Figure 1. These data are compared with the model prediction discussed in the next Sect. Important to note, in both cases these counts show a maximum in flux density and then a rather quick convergence towards faint fluxes. This is evidence that the total EBL at the two wavelengths is mostly resolved at the limiting fluxes of the deepest surveys (see Madau \& Pozzetti 2000 for a similar consideration about the optical counts).

Since we are interested not only in the local EBL intensity, but also in its time evolution, we need information on the source spatial density as a function of redshift. Various flux-limited Spitzer samples have been used for deriving redshift surveys with good degrees of completeness, suitable for the calculation of redshift-dependent luminosity functions. Rodighiero et al. (2010) have worked on a complete $24 \mu \mathrm{m}$ selected sample, while Magnelli et al. (2009) have done it on a sample selected with MIPS at $70 \mu \mathrm{m}$. The two surveys have been used to calculate galaxy 
luminosity functions at the rest-frame wavelengths of 15 and $35 \mu \mathrm{m}$, shorter than the selection wavelengths to minimize the K-corrections for the typical source redshift $(z \sim 1)$.

We report the two redshift-dependent luminosity functions in Figures 2 and 3, together with the predictions of our evolutionary model in Sect. 3.

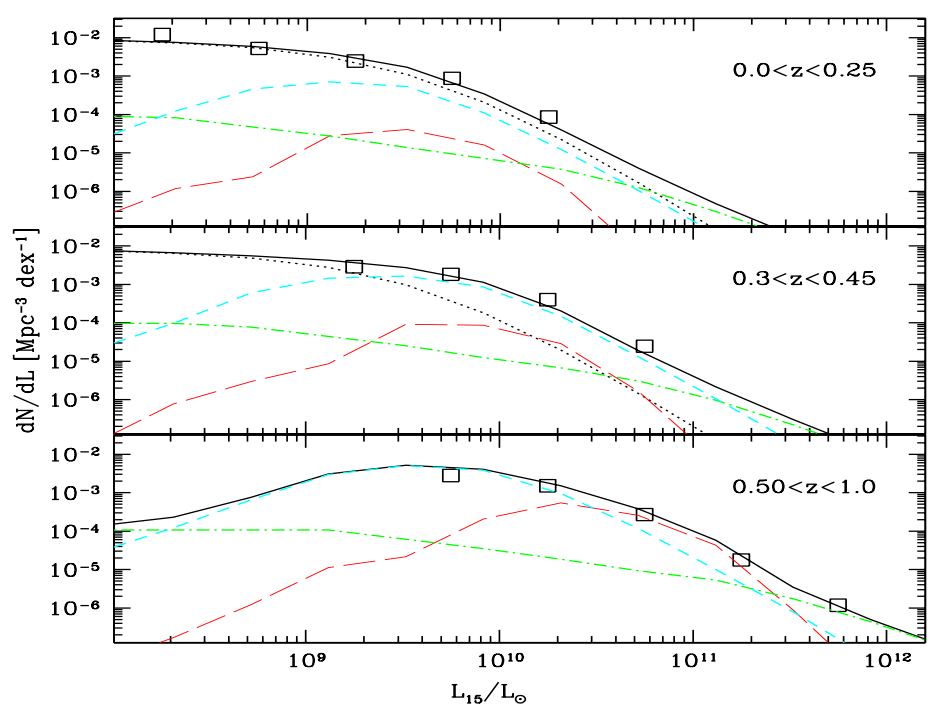

Figure 2: The rest-frame $15 \mu \mathrm{m}$ luminosity functions of IR-selected galaxies in redshift bins, in comoving units. The data-points are from an Spitzer-MIPS selected sample at $24 \mu \mathrm{m}$ by Rodighiero et al. (2010), computed at $15 \mu \mathrm{m}$ to minimize the K-corrections. Green dot-dashed line: type-I AGNs. Cyan short-dashed line: evolving moderate-luminosity star-forming galaxies (LIRG). Red long-dashed line: high-luminosity starbursts (ULIRG). Lower dotted black line: quiescent spiral population. The upper black continuous line is the total predicted emissivity.

\subsection{The Herschel multi-wavelength number counts and luminosity functions}

The major new step of the recent years at the long far-IR and sub-mm wavelengths has been offered by the ESA Herschel space observatory, whose imaging instruments, PACS and SPIRE, have surveyed deeply and extensively at the wavelengths of 70,100, $160 \mu \mathrm{m}$ and 250, 350, 500 $\mu \mathrm{m}$, respectively. Of specific interest for us, the PACS observations (Berta et al. 2010) have exploited the diffraction-limited imaging capability of the instrument to reduce the confusion noise and ease source deblending and identification. PACS imaging requires however long integrations and has been performed to such faint fluxes only in small areas, like GOODS and COSMOS. We report in Figure 1 faint number count data at the effective wavelength of $70 \mu \mathrm{m}$ from Berta et al. (2011), which account for all various corrections of sampling completeness, effective area, fraction of spurious identifications, together with previous determinations by Bethermin et al. (2010) based on Spitzer observations.

The Herschel extensive cosmological surveys have also offered us the opportunity for calculating redshift-dependent luminosity functions in the far-IR and sub-millimeter. Herschel SPIRE data over a total of $38.9 \mathrm{deg}^{2}$ within five high-latitude fields have been used by Marchetti et al. (2014), to compute the local luminosity functions in the sub-millimeter at $250 / 350 / 500 \mu \mathrm{m}$, and 


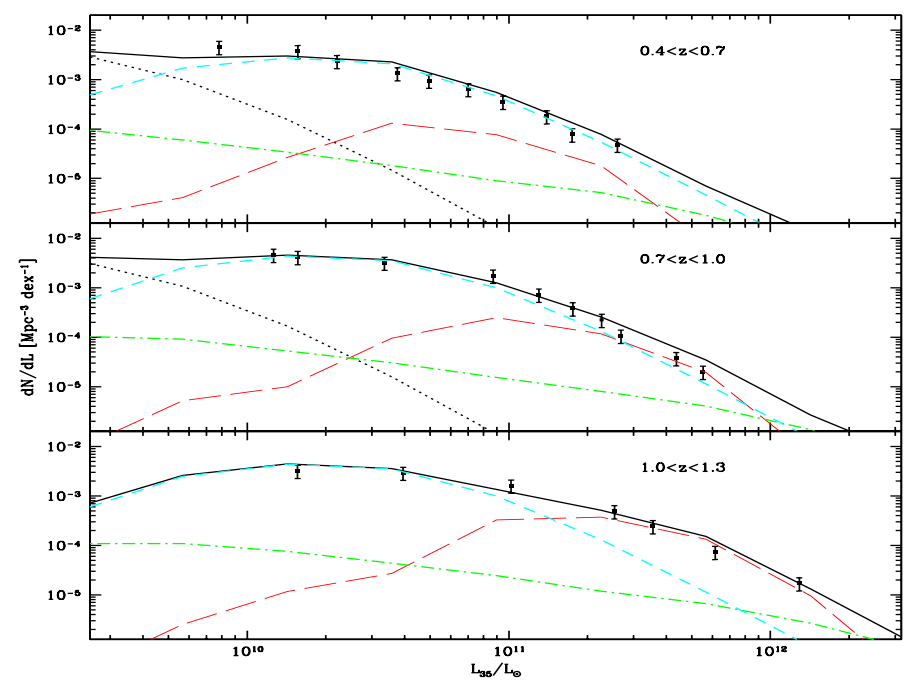

Figure 3: The rest-frame $15 \mu \mathrm{m}$ luminosity functions of IR-selected galaxies in redshift bins, in comoving units. The data-points are from a Spitzer-MIPS selected sample at $24 \mu \mathrm{m}$ by Rodighiero et al. (2010), computed at $15 \mu \mathrm{m}$ to minimize the K-corrections. Green dot-dashed line: type-I AGNs. Cyan short-dashed line: evolving moderate-luminosity star-forming galaxies (LIRG). Red long-dashed line: high-luminosity starbursts (ULIRG). Lower dotted black line: quiescent spiral population. The upper black continuous line is the total predicted emissivity.

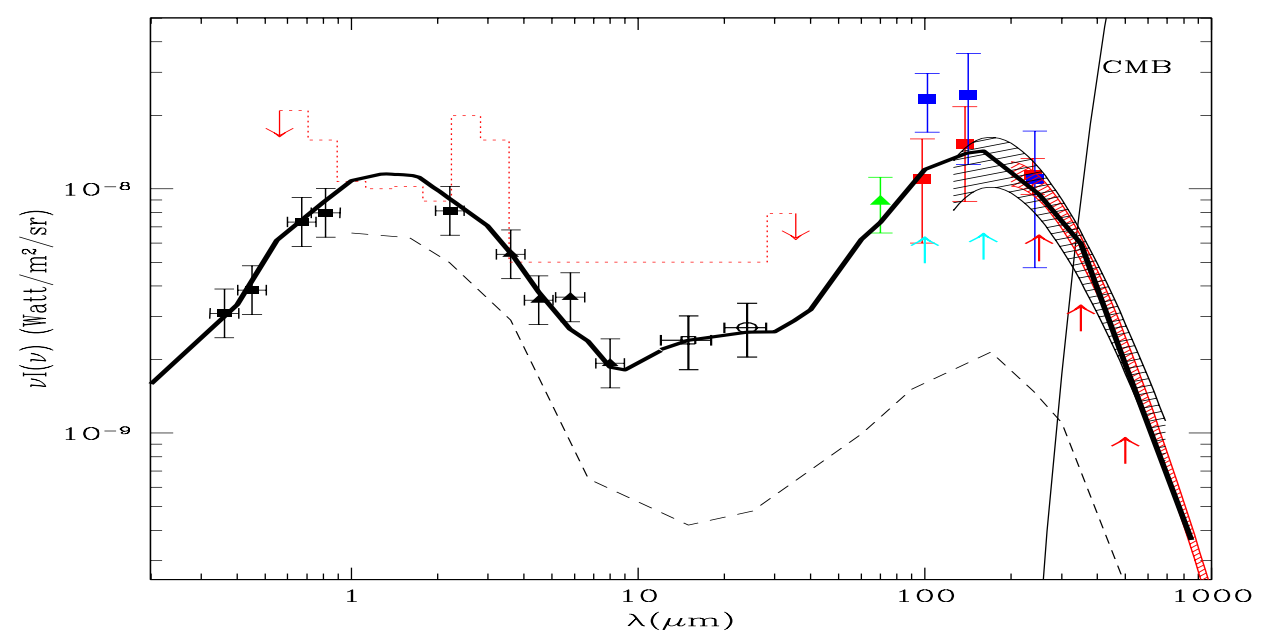

Figure 4: Comparison of data on the CIRB intensity at long wavelengths and our best-fit model predictions (thick line). Most of the data are as reported in AF2008. The cyan arrowheads mark the fraction of the CIRB intensity resolved into sources by Herschel PACS (Berta et al. 2010, 2011) at 100 and $160 \mu \mathrm{m}$, a fraction corresponding to roughly half of the COBE intensity at these peak wavelengths. Red arrowheads at longer wavelengths mark the CIRB resolved fractions with Herschel SPIRE (Oliver et al. 2010, Glenn et al. 2010).

in the far-IR, in combination with Spitzer photometry and archival data (including redshifts from SDSS). This provides us with a census of the luminosity density in the local Universe at these 
wavelengths.

Gruppioni et al. (2013) and Vaccari et al. (2015) report independent multi-wavelength luminosity functions, from the rest-frame 60 to $500 \mu \mathrm{m}$, over a wide redshift interval from local to $z \simeq 4$.

\section{EBL model improvements and the cosmic $\gamma \gamma$ opacity}

We have accumulated and included in our AF2008 modeling only new data at long wavelengths, $\lambda>10 \mu \mathrm{m}$.

The recent data from the Herschel surveys have slightly modified previous analyses based on the Spitzer observations. The latter required some substantial extrapolations from the observed mid-IR to long wavelengths, that are not confirmed by the new far-IR data. Elbaz et al. (2010) and Rodighiero et al. (2010) found, in particular, that the highest luminosity galaxies over a wide redshift interval have a relatively lower IR luminosity with respect to previous estimates. Consequently, both the estimated contribution of sources to the EBL intensity at IR wavelengths and the photonic number densities at high redshifts are slightly decreased with respect to reports by AF2008.

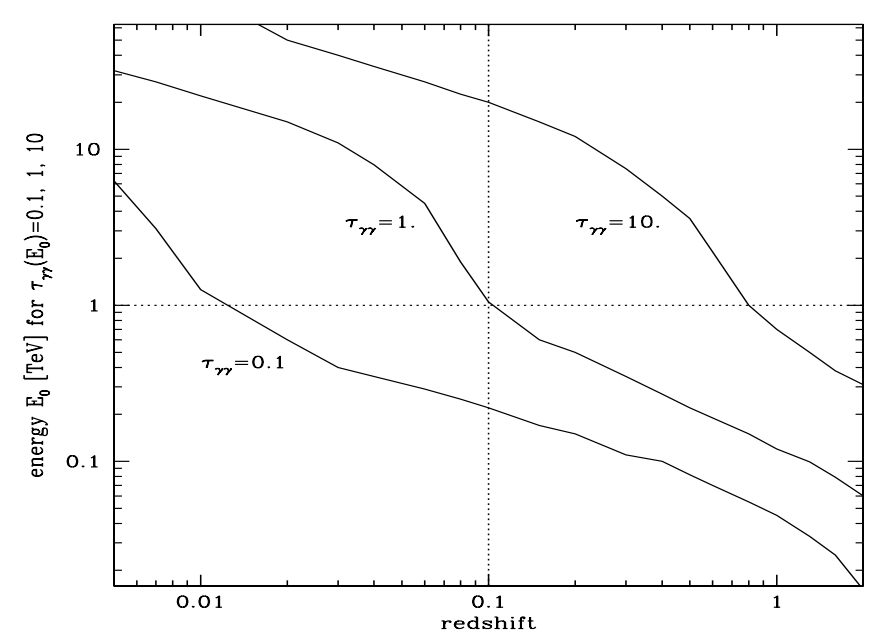

Figure 5: The energies corresponding to optical depth values of $\tau=0.1,1$ and 10 for photon-photon collisions, as a function of the redshift distance of the source.

Figure 4 reports our current assessment of the local EBL intensity after the above mentioned corrections. We see that all the spectral range from the UV to $8 \mu \mathrm{m}$ has remained unchanged as a consequence of the unchanged modeling of the galaxy populations at these frequencies. Instead, the mid-IR portion from 10 to $40 \mu \mathrm{m}$ has progressively lowered because of the slightly reduced evolution of IR emissivity of galaxies. And the whole far-IR and sub-millimetric peak of dust emission from galaxies has correspondingly lowered: the peak EBL emission at $160 \mu \mathrm{m}$, while estimated to exceed $20 \mathrm{nW} / \mathrm{m}^{2} / \mathrm{sr}$ by AF2008 based on Spitzer data, is now lowered to $\sim 14 \mathrm{nW} / \mathrm{m}^{2} / \mathrm{sr}$. 
This modification of the EBL intensity and its evolution impacts only on very high energy $\mathrm{TeV}$ observations of essentially local cosmic sources, according to the usual rule-of-thumb

$$
\lambda_{\max } \simeq 1.24\left(E_{\gamma}[\mathrm{TeV}]\right) \mu m .
$$

so a significant influence might only be for the observations of BLAZAR spectra at several to few tens of $\mathrm{TeV}$.

The scaling of the optical depth for photon-photon interaction as a function of redshift and energy are reported in Figure 5, which reveals a slight modification with respect to AF2008 in the sense of a modest increase of the redshift corresponding to an optical depth of $\tau_{\gamma \gamma}=1$ and 10 . We refer to AF2008 for all details concerning the calculations.

\section{Discussion and Conclusions}

Compared with the results in AF2008, the new data on the IR source emissivities at long IR wavelengths, and the corresponding slightly lower EBL intensity values, imply somewhat reduced photon-photon opacities at the highest photon energies at $>1 \mathrm{TeV}$. The difference is not large, and involves only the highest energy observations. This typically mostly concerns absorption effects in the most local objects, like the classical MKN 501 and MKN 421.
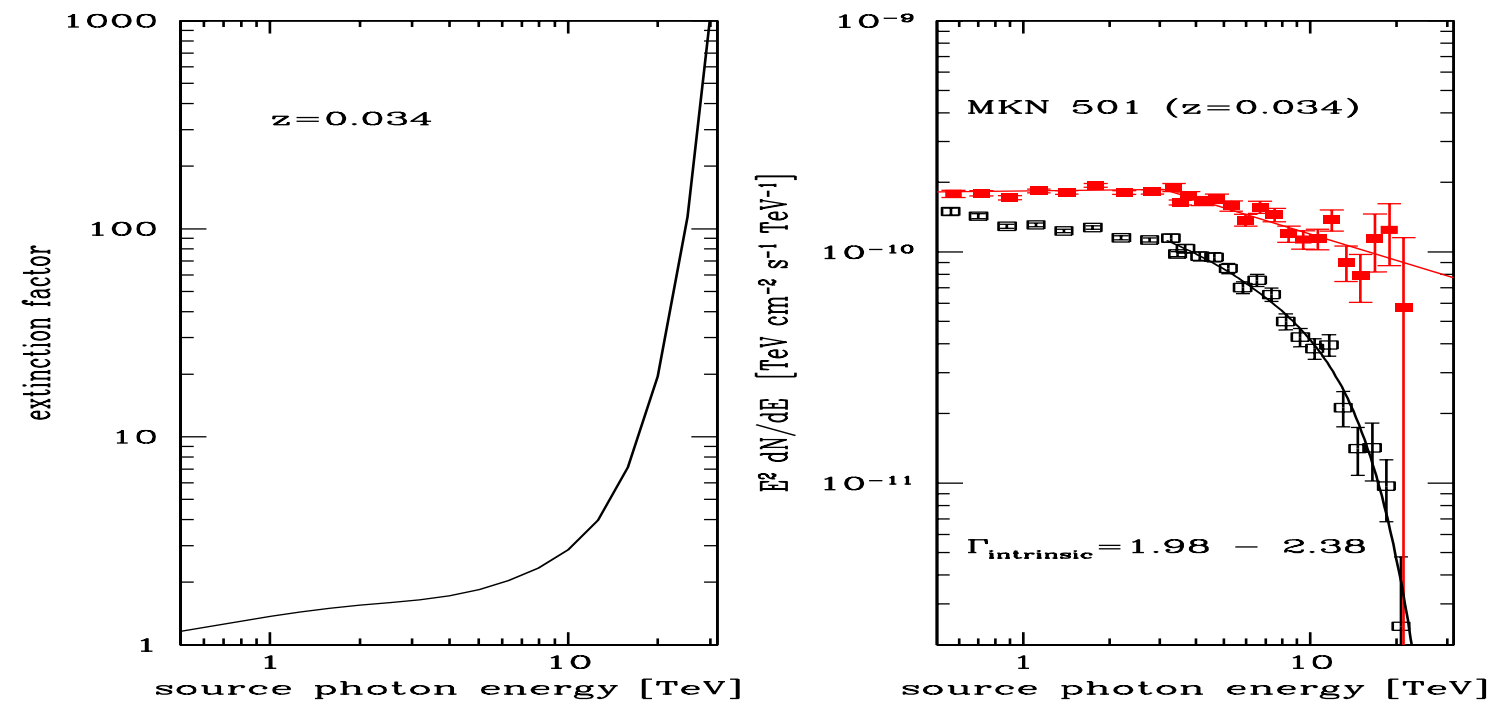

Figure 6: Left: Absorption correction for the source MKN 501 at $z=0.034$. Right: the observed (open black) and absorption-corrected (filled red) spectrum. Data from Aharonian et al. (2001).

For illustration purposes, we report in Figure 6 our detailed estimate of the pair-production extinction factor for a source distance of $\mathrm{z}=0.034$, corresponding to that of $\mathrm{MKN} 501$. On the right panel of the figure we show the observed and corrected spectra. Based on our extinction correction, the intrinsic spectrum is well reproduced by two-power-laws, one with photon spectral index of $\sim 2$ up to $3 \mathrm{TeV}$, followed by a steeper one with index 2.4 above. The high-energy upturn in the corrected spectrum indicated in the Costamante (2012) review is not apparent here. Our current 
results are in line with those reported as fiducial model in Primack et al. (2011). In our case, the modeling of the extragalactic source contribution to the EBL is purely empirical, and based on an enormous variety of deep photometric imaging data and accurate rendition of the source spatial distributions, like detailed a knowledge of the source luminosity functions at all wavelengths from UV to the sub-millimeter.

Overall, our improved EBL modeling reduces some tension between VHE observations of BLAZAR and standard physical interpretations of their spectra, once appropriate account is taken of pair-production effects generated by photon encounters.

This subject about the interaction of the highest energy photons produced by cosmic sources and the background light will likely remain a hot topic in the coming years. Proof of this is, among others, the recent claim by Zemcov et al. (2014) about an observed excess signal of near-IR background fluctuations on scales of about 10 arcminutes that may correspond to diffuse light from the intergalactic space outside galaxies, potentially implying an EBL flux two times higher, from the optical to the near-IR, than our estimated galaxy contribution in Fig. 4. How much would this be consistent with the observed BLAZAR spectra will certainly be considered in forthcoming publications.

\section{References}

[1] Ackermann, M., Ajello, M., Allafort, A., et al., 2012, Science 338, 1190

[2] Aharonian, F., et al., 2006, Nature, 440, 1018

[3] Aharonian, F.A., et al., 2001, A\&A 366, 62

[4] Albert, J., Aliu, E., Anderhub, H., et al., 2006, ApJ 642, L119

[5] Berta, S., et al., 2010, A\&A 518, L30

[6] Berta, S., Magnelli, B., Nordon, R., et al., 2011, A\&A 532, 49

[7] Costamante, L., 2012, in The Spectral Energy Distribution of Galaxies, Proceedings IAU Symposium No. 284, 2011, R.J. Tuffs \& C.C.Popescu, eds. (astro-ph:1204.6426v1)

[8] de Angelis, A., Roncadelli, M., Mansutti, O., 2007, PhRvD 76, 1301

[9] de Angelis, A., Mansutti, O., Persic, M., Roncadelli, M., 2009, MNRAS 394, L21

[10] Dwek, E., \& Slavin, J. 1994, ApJ, 436, 696

[11] Elbaz, D., Hwang, H. S., Magnelli, B., et al, 2010, A\&A 518, L29

[12] Franceschini, A., Rodighiero, G., Vaccari, M., 2008, A\&A 487, 837

[13] Gilmore, Rudy C., Somerville, Rachel S., Primack, Joel R., Dominguez, A., 2012, MNRAS 422, 3189

[14] Glenn, J., Conley, A., Bethermin, M., et al., 2010, MNRAS 409, 109

[15] Gould, R. J., \& SchreÂt'der, G. 1966, Phys. Rev. Lett., 16, 252

[16] Jaeckel, J., A. Ringwald, Ann. Rev. Nucl. Part. Sci., 2010, 60, 405

[17] Nikishov, A. I. 1962, Sov. Phys. J. Exp. Theor. Phys., 14, 393

[18] Oliver, S. J.; Wang, L.; Smith, A. J., et al., 2010, A\&A 518, L21O 
[19] Persic, M., de Angelis, A., 2008, A\&A 483, 1

[20] Primack, J. R., et al., 2011, Extragalactic Background Light and Gamma-Ray Attenuation, in American Institute of Physics Conference Series, eds. F. A. Aharonian et al., American Institute of Physics Conference Series, Vol. 1381, pp. 72âĂŞ83. arXiv:1107.2566.

[21] Rodighiero, G., Cimatti, A., Gruppioni, C., et al., 2010, A\&A 518, L25

[22] Stanev, T., \& Franceschini, A. 1998, ApJ, 494, L159

[23] Stecker, F. W. 1969, ApJ, 157, 507

[24] Stecker, F. W., de Jager, O. C., \& Salamon, M. H. 1992, ApJ 390, L49

[25] Stecker, F. W., Malkan, M.A., Scully, S.T., 2006, ApJ 648, 774

[26] Tavecchio, F., Roncadelli, M., Galanti, G., Bonnoli, G., 2012, PhRvD 86, 5036

[27] Zemcov, M., et al., 2014, Science, 346, 6210, 732. 